Andrews University

Digital Commons @ Andrews University

Honors Theses

Undergraduate Research

$11-26-2018$

\title{
Investigating the Effects of Popularity on Comment Civility: A YouTube Case Study
}

Isaac Smith

Andrews University, ismith@andrews.edu

Follow this and additional works at: https://digitalcommons.andrews.edu/honors

Part of the Communication Commons

\section{Recommended Citation}

Smith, Isaac, "Investigating the Effects of Popularity on Comment Civility: A YouTube Case Study" (2018). Honors Theses. 214.

https://dx.doi.org/10.32597/honors/214

https://digitalcommons.andrews.edu/honors/214

This Honors Thesis is brought to you for free and open access by the Undergraduate Research at Digital Commons @ Andrews University. It has been accepted for inclusion in Honors Theses by an authorized administrator of Digital Commons @ Andrews University. For more information, please contact repository@andrews.edu. 


\section{J. N. Andrews Honors Program \\ Andrews University}

\section{HONS 497}

Honors Thesis

Investigating the Effects of Popularity on Comment Civility: A YouTube Case Study

Isaac Smith

$11 / 26 / 2018$

Advisor: Dr. Heather Day

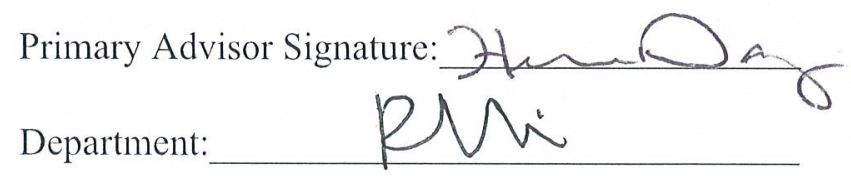




\section{J. N. Andrews Honors Program \\ Andrews University}

\section{HONS 497}

Honors Thesis

Investigating the Effects of Popularity on Comment Civility: A YouTube Case Study

Isaac Smith

$11 / 26 / 2018$

Advisor: Dr. Heather Day

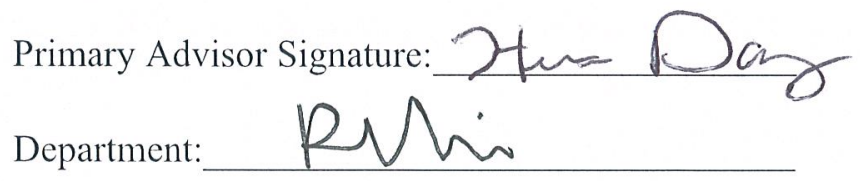




\section{Abstract:}

This study will explore the connection between the civility of internet comments and their popularity- through the example of two YouTube videos. By using a combination of manual review and computer analysis, to help readers discover if video popularity has a negative effect on comment civility. Findings also examined correlations between factors that indicate civility, while also including examples of notable and intriguing comments found during this study.

\section{Introduction:}

Han et al. (2018) claims that civil discussion is necessary for the continuation of democracy, and a large number of communication scholars are trying to cultivate a greater understanding of civil discussion and how to promote it. In addition to general discussion, many formal studies and papers have been produced recently on the topic of online comments- this work is becoming a growing segment of communication research.

Most comment research centers around social media, and one of the largest and most influential social media sites in existence is YouTube. Currently, the platform has 1.8 billion registered users, and is second only to Facebook in size. It is one of the largest public forums to ever exist, nigh unparalleled in its diversity of content. YouTube's comment section has gained a notorious reputation for offensive behavior, as articulated by Dhiraj and Sanjay, 2018.

The researcher is interested in pursuing similar research to Han et al. and Dhiraj and Sanjay, using textual analysis to explore deeper into the nature and logic behind comment civility. Han et al. focused on the effects of modeling in online communities. Dhiraj and Sanjay investigated how various commenters interacted with each other across several videos. The methodology and purpose of this study is similar to those examples, but samples for analysis were decided from statistical factors.

There seems to be a tendency towards discourse being less and less civil the more popular someone's video becomes. This tendency is defined as The Law of Big Numbers (Dhiraj and Sanjay, 2018). So much content is being uploaded to YouTube that it's impossible for moderators to police and regulate the discussion. According to this theory, a larger video or channel will receive more comments and be more difficult to moderate as a result.

The researcher performed a comparative analysis between the comment sections of two videos. The videos were about the same subject, and similar in content- the main difference being in popularity. (Fig. 1) The hypothesis, according to the Law of Big Numbers, is that the video with the larger view count will have less civil comments compared to the video with the smaller view count.

Before we can discuss which comments qualify as civil, we need to first define civility in the context of online discussion. Han et al (2018) provides a general understanding by focusing on the presence of politeness and support towards people and ideas that you disagree with. Comments of support, affirmation, and understanding, in short. After all, it is easy and natural to support what you 
factors to evaluate the comments on and using numerical scores to portray the presence or absence of those factors. (Fig. 2)

For this study, the researcher compared and analyzed the user comments on two different videos. To prevent the statistical information from becoming outdated and for ease of use, the comments were evaluated using .CSV spreadsheets produced by Philip Klostermann's YouTube Comment Scraper. (Fig. 3) YCS is a free, open source website that allows the user to easily gather comments, replies, and date/timestamps from a certain YouTube video, and ultimately gather large quantities of information automatically for evaluation.

To use YCS, paste the URL of the video you want to scrape comments from, and change the settings to match your interests before running the program. For this project the researcher chose to receive the name of the commenter, the comment's timestamp, the text of the comment, replies to a comment, and the number of likes each comment has. After the program has completed the data collection, save the data as a .CSV spreadsheet. All of the comments on both videos as of Sept. 18 were archived, and were used for the analysis.

These .CSV spreadsheets were statistically analyzed using the LIWC software, under the advice of Dr. Bailey. Before analysis, users need to correct spelling errors and remove special characters/emoticons from the comments so LIWC can analyze the information effectively. Also, it's recommended to convert the .CSV file to a .xlsx spreadsheet, which is easily done in Excel or Google Sheets. To analyze the comment information, ensure that the comment text to be analyzed is in a single spreadsheet column, select that column in LIWC, and hit analyze. The program will put the statistical output for each cell on the same row, next to the original text it was derived from.

In addition to LIWC's built-in dictionary, the software easily accommodates the creation and use of custom dictionaries to reflect language and expressions used by specific groups. LIWC supports input from Word, Excel, and .CSV spreadsheet files, and can save results in the aforementioned formats to accommodate a variety of statistical programs. The output variables are incrementally increased to reflect the usage of specific keywords that fit into a variety of categories, providing in-depth statistics to facilitate 'manual' investigations and judgment.

Because both videos are about the same game and are both walkthroughs, the biggest difference between the two should be the popularity of each video. The primary hypothesis is that the smaller community (theSeraphim17's) will have a more civil comments section in comparison to theRadBrad's. In the case of theSeraphim17's video, the number of comments is small enough that the researcher can simply examine and review each one individually.

Given that theRadBrad's video had over 25 thousand comments at the time of this study, a representative random sample of approximately 400 comments was chosen to look at $0.016 \%$, for review. That number will provide a $95 \%$ confidence level and a confidence interval of 5 , considering the size of the original population. The listing of comments was then been divided into 20 segments, to ensure more equitable sampling, and 20 comments were then selected at random from each segment for a total of 400 . 
The fourth factor is if the comment is noticeably showing respect to the Original Poster or to the video's content. This was determined by a high score in the LIWC categories of 'authentic' and 'emotional tone'/ 'positive emotion'. A normal comment receives a 0 . Something along the lines of 'I really like your stuff' or 'Those guys are great' would receive a 1 . People that are respectful to other individuals is a sign of civil discussion.

And finally, all comments and other visible activity by the Original Poster will receive special attention because they don't fit easily into the other categories. We might not be able to see the work that the OP does behind the scenes, but we can see how much public interaction they have with their commenters.

\section{Results:}

The data gathered was mostly inconclusive, and does not support or refute the hypothesis that popular videos are more incivil than smaller videos. Emotional Tone and Authenticity were the only two categories that showed statistically significant results. (Fig. 4.) RadBrad's comments scored higher on both of these positive categories. It is probable that this is because his commenters are directly reflecting their positivity and happiness through direct language that's easily picked up by the program. Also, any malicious behavior in both comment sections is clearly in the minority, so there are no substantial differences on that front.

The rest of the T-test comparisons weren't statistically significant or reliable, considering the subjectivity of comparing written words and the difference in sample sizes. There are several possible reasons for these results. One is that the sample size of two videos may be too small to examine any larger trends or notice subtle differences in interactions between the two examples. Perhaps the study should have been conducted on two videos with a more controversial topic, then results could have shown more differences between each comment sections.

The researcher's decision to score the custom factors as 0 or 1 likely made it more difficult to conduct a T-test analysis for those factors, because T-tests aren't designed to evaluate binary input. For continued research, a numerical scale (between 1-10 for example) would provide more easily analyzed data.

The secondary hypothesis of positive correlation between positive factors was also unproven, thanks to a general lack of statistically significant results to compare. A recommendation for future researchers would be to focus on a single hypothesis and a small number of factors to avoid an unwanted escalation of scope during the process of research.

There were substantial differences in the data that couldn't be analyzed by LIWC. TheRadBrad's video had a lot more emojis and mistakes in spelling and punctuation, but those things don't make his comment section less civil. While one could argue that the nature of those comments lowers the quality of the discussion, that issue is a diversion from the original hypothesis.. 
The expression of civility in modeled replies to offensive behavior was of substantial interest, thanks to being directly quantifiable and relating to the concept of Meso Space in a comment discussion. Also, the presence of such replies indicates the existence of a sense of community and community standards that are meant to be upheld, reflecting the presence of civility in a direct manner. If the researcher had opportunities for future research, they would be interested in investigating specific examples of such modeled replies in a variety of contexts.

Through a greater understanding of civility and it's expression, it is possible to study and determine the best ways to inspire civil behavior. These methods can then be applied in our own communities and interactions, for the sake of encouraging civil discourse and general wellbeing. But it is important to remember that any community is made up of a number of different people, who each have individual wants and purposes. The community or comments section is only as good or bad as the people involved make it.

Han et al (2018) found confirmation that modeled responses had a positive effect on community behavior, and Dhiraj and Sanjay traced the actions of individuals over time and across a community of videos. In this researcher's opinion, future research could benefit from a synthesis of the two approaches1) investigating and 2) tracing individuals who make large quantities of modeled responses on social media. Several questions could be investigated, such as 'what things do these people have in common?' and 'what groups or communities do they ascribe themselves to?' Discovering more about these individuals who nourish civility is of utmost import to expanding the understanding of social media. 
overall quality of the situation. In addition to providing an example of statistically measuring and evaluating civility, it's results about the usefulness of moderation and metacommunication are extremely useful to support my own hypothesis. I believe that a smaller channel is more likely to experience metacommunication among its members, and that modeling will be more effective in those communities.

Reader, B. Free Press vs. Free Speech? The Rhetoric of "Civility" in Regard to Anonymous Online Comments. Journalism \& Mass Communication Quarterly. 2012, Vol 89, Issue 3, pp. 495 - 513. https://doi.org/10.1177/1077699012447923

Reader examines the purpose and value of anonymity in online discussion, using the 'concordance' framework to analyze the point of view of high-profile journalists versus that of online commenters who responded to the journalists. He explains that the journalists dehumanized and claimed that anonymous opinions are worthless and repugnant, and by nature incivil. In response, the majority of commenters supported anonymous commentation, claiming that it provides privacy and protection so people can voice their opinions and provide valuable and necessary critique. Reader ultimately suspects that the main issue of divisiveness arises from the subjective definition of civility, and that more research is required to prove that anonymity causes incivility. I feel that this exploration of the divide between content producers (in this case, experienced journalists) and commenters helps us understand why many researchers and academics don't study many forums of communication- they see the discussion that goes on there as not worthy of their time. I will need to keep in mind that the line between civility and incivility is often narrow, and be careful not to judge too harshly because of some perceived moral high ground.

Pennebaker, J.W., Boyd, R.L., Jordan, K., \& Blackburn, K. (2015). The development and psychometric properties of LIWC2015. Austin, TX: University of Texas at Austin. DOI: 10.15781/T29G6Z http://liwc.wpengine.com/wp-content/uploads/2015/11/LIWC2015 LanguageManual.pdf http://liwc.wpengine.com/wp-content/uploads/2015/11/LIWC2015 OperatorManual.pdf

Pennebaker, et al. developed the LIWC software to efficiently study and measure the emotional and structural components in samples of writing. LIWC is primarily a tool for psychometric analysis, examining large quantities of text for the presence It is an incredibly versatile and customizable software, using a customizable 'dictionary' (developed and evaluated by a committee of qualitative judges) that searches for words and word stems that fit into a wide range of significant categories- like 'power words', 'informal language', and 'quantifiers.' The dictionary can also be customized, to add specific words or terms unique to a certain field or discipline. I'm incredibly excited about the possibilities that LIWC provides, as it's specific statistical categories will make it far easier to evaluate the large quantities of information I'm working with.

Appendix:

Fig. 1: Video Size. 


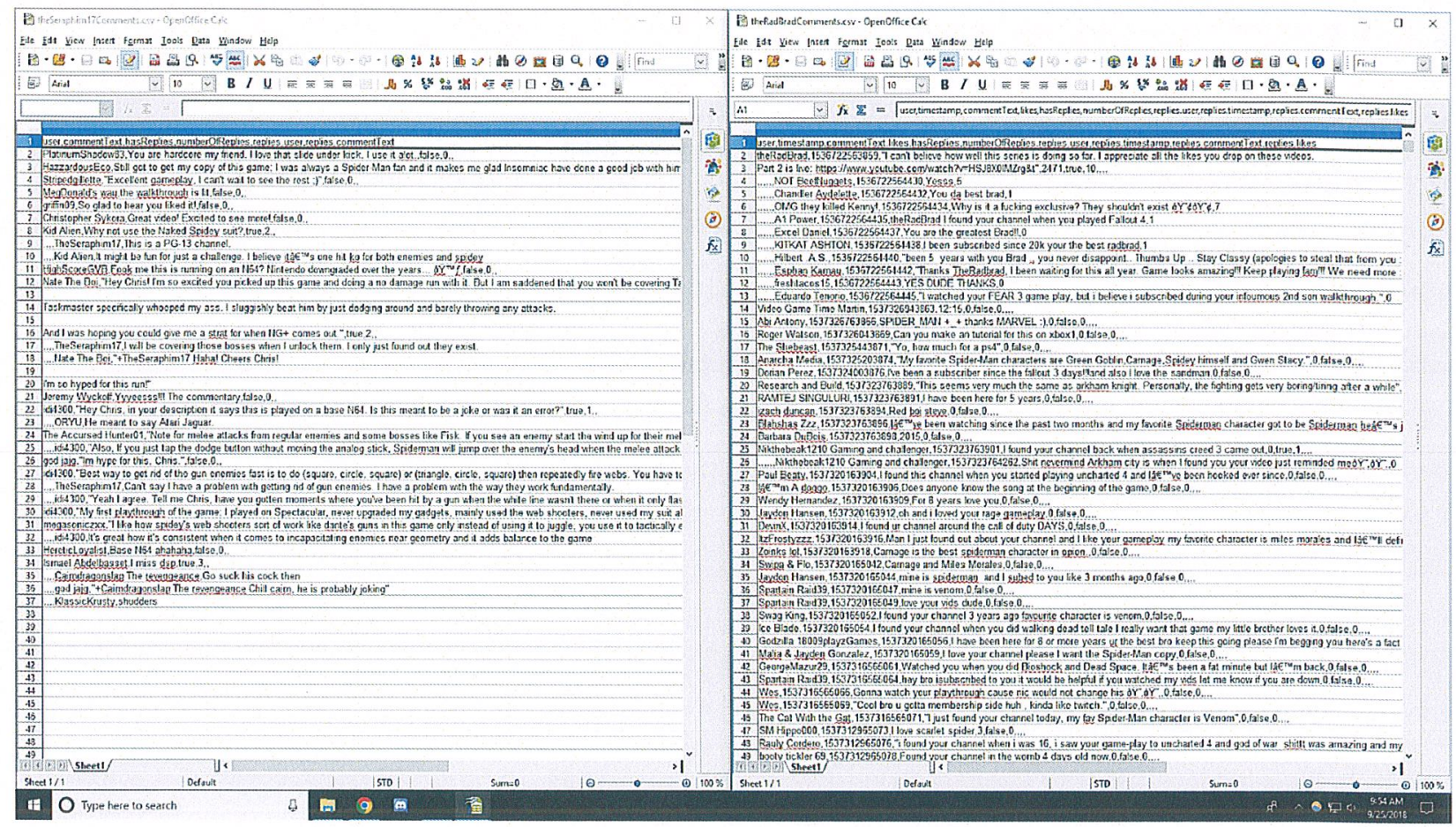

Fig. 4: LIWC Results.

\begin{tabular}{|l|l|l|l|l|}
\hline & P-value & Cohen's D & Radbrad Mean/Average & Seraphim Mean/Average \\
\hline Tone: & 0.014 & 0.4924 & 62.952 & 44.18 \\
\hline Authenticity: & 0.0003 & 0.5601 & 54.399 & 31.81 \\
\hline
\end{tabular}

Independent Samples T-Test

\begin{tabular}{llrllrc}
\hline & & statistic & df & $\mathbf{p}$ & Mean difference & Cohen's d \\
\hline anger & Welch's t & -1.705 & 33.7 & 0.097 & -1.443 & -0.3097 \\
$\underline{\text { Tone }}$ & Welch's t & 2.591 & 33.2 & $\underline{0.014}$ & $\underline{18.770}$ & $\underline{0.4924}$ \\
Clout & Welch's t & 1.371 & 33.3 & 0.180 & 8.721 & 0.2602 \\
Analytic & Welch's t & -1.188 & 33.0 & 0.243 & -8.280 & -0.2305 \\
Authentic & Welch's t & 3.231 & 34.3 & $\underline{0.003}$ & $\underline{22.586}$ & $\underline{0.5601}$ \\
posemo & Welch's t & 1.201 & 37.2 & 0.237 & 2.029 & 0.1740 \\
WC & Welch's t & -1.248 & 30.7 & 0.221 & -4.547 & -0.3452 \\
WPS & Welch's t & 0.521 & 35.0 & 0.606 & 0.792 & 0.0856 \\
Sixltr & Welch's t & -0.783 & 30.8 & 0.440 & -3.704 & -0.2119 \\
\hline
\end{tabular}

